\title{
Producción científica sobre temas educativos y formativos en comunicación, información y documentación en las revistas de documentación españolas (2000-2019)
}

\author{
Scientific production on Communication, Information and Documentation \\ educational subjects in Spanish LIS journals (2000-2019)
}

\section{Juan Carlos Marcos Recio (1), Juan Miguel Sánchez Vigil (2), María Olivera Zadlua (2)))}

(1) Facultad de Ciencias de la Información de la UCM, Av. Complutense s/n 280401, jmarcos@ucm.es.

(2) Facultad de Ciencias de la Documentación de la UCM, C/Satntísima Trinidad 37 28010, jmvgil@ucm.es, molivera@ucm.es

\section{Resumen}

Se analiza la producción científica sobre temas educativos y formativos en materia de Comunicación, Información y Documentación en las principales revistas de Documentación españolas durante los últimos veinte años (2000-2019), con el fin de obtener información sobre autoría, contenidos y temáticas específicas. Los trabajos son escasos, resultando 231 artículos de 336 autores pertenecientes a 99 instituciones públicas y privadas, en los que se analizan contenidos centrados en una docena de materias generales. Se concluye, a partir de la escasez de artículos, en la necesidad de profundizar en el tema objeto de estudio, y de prestar una mayor atención por parte de los editores a la normalización y selección de descriptores o palabras clave de los artículos, con el fin de facilitar una mayor y mejor recuperación de la información.

Palabras clave: Educación. Formación. Docencia. Comunicación. Información. Documentación. Producción científica. Revistas de documentación. España.

\section{Introducción}

La producción científica sobre la educación, en determinadas áreas, sigue siendo un enigma difícil de resolver, sobre todo en disciplinas tan transversales como lo son la Comunicación, la Información y la Documentación. Excepto las materias que tienen un recorrido histórico (Eco, 2015), como el Trivium (gramática, retórica y dialéctica) y el Quadrivium (aritmética, geometría, astronomía y música), el resto han tardado varios siglos en asentarse como disciplinas importantes. Las corrientes actuales tratan de unificar, de integrar los estudios como se hizo en épocas pasadas, pero se viven tiempos colaborativos en la sociedad y en la educación. Por este motivo, la transversalidad de la docencia requiere de otras áreas que también aportan recursos y valores a la Educación, en este

\begin{abstract}
The scientific production on educational and formative subjects regarding Communication, Information and Documentation in the leading LIS journals over the past twenty years (2000-2019) is analysed, in order to obtain information on their authorship, contents and specific subject matter. It was discovered that there have been relatively few studies carried out, resulting in 231 articles from 336 authors belonging to 99 public and private institutions, and contents focused on a dozen of general topics. The leading conclusion reached is the scarcity of articles and therefore the need to delve more deeply into this field. Furthermore, the publishers should devote greater attention to the standardization and selection of descriptive or key words in the article, to promote an increased, improved and easier retrieval of information on the subject.
\end{abstract}

Keywords: Education. Training. Teaching. Communication. Information. Documentation. Scientific production. LIS journals. Spain.

caso a través de las publicaciones de Documentación, Información y ciencias afines.

Mientras una parte importante de los docentes e instituciones científicas consideran que la producción científica es fundamental para complementar la formación (Nóvoa \& DeJong, 2003; UNESCO, 2004; Beaver, 2001; CONACYT, 2006; Laval \& Weber, 2002; Harter \& Hooten, 1992), otros apuestan por la practicidad de los estudios para enfrentarse a los retos de la ciencia (Perrenoud, 1998; Labaree, 2000; Osorio, 2012) y tienen como valor supremo la calidad de la educación en Europa, así como la internacionalización y globalización de los estudios universitarios (Van Vught, 1992; Altbach \& Teichler, 2001).

Con carácter general es en el área de Bibiblioteconomía y Documentación donde se ha realizado 
numerosos estudios, tanto en el caso español (Fillipo, 2013) como en el de Latinoamérica, especialmente México y Brasil (Herrero \& Ríos, 2006; Gómez, 1993; Licea \& Valles \& Arévalo \& Cervantes, 2000; Marteleto, 1998) o con una visión más internacional (Moya, \& Herrero, 2002; Larivière \& Lebel \& Lemelin, 2004); sobre todo en el estudio de la producción científica en revistas del área, pero también en visibilidad internacional e investigación colaborativa. Estos logros se han establecido gracias al esfuerzo de equipos de investigación a través de universidades y centros de investigación e instituciones educativas que actúan en defensa de la ciencia y su visibilidad (González Uceda, 1997).

Como se verá en el estudio, estos planteamientos requieren de un esfuerzo por parte de la comunidad docente para dar más valor a las publicaciones del área de la documentación. Los resultados aportados vienen a demostrar que hay un camino por explorar, que se abren nuevas rutas, que otras estrategias también son válidas y que el futuro las demandará.

Los resultados de los estudios sobre educación y formación generalmente se difunden en revistas vinculadas a esas materias específicas, por lo que los trabajos en publicaciones de otras áreas tienen menor difusión o visibilidad, como es el caso de la Documentación. Es objeto de este artículo analizar la producción científica sobre temas educativos y formativos en materia de Comunicación, Información y Documentación en las principales revistas de Documentación en España durante los últimos veinte años (2000-
2019), con el fin de obtener información sobre autoría, contenidos y temáticas específicas.

Partiendo de estas premisas se ha llevado a cabo una revisión general del estado de la cuestión y de las fuentes. En relación con las revistas seleccionadas para este estudio se han considerado las referencias generales de Guallar, Ferrán, Abadal y Server (2017) en "Revistas científicas de información y documentación: Análisis temático y metodológico"; y de manera concreta los trabajos sobre la Revista Española de Documentación Científica (Ríos, 2001), Revista General de información y Documentación (López \& otros, 2001), o Documentación de las Ciencias de la Información (Arquero \& Río, 2002). Sobre la producción general en la disciplina Documentación se han obtenidos datos de Delgado (2001), Arquero (2002, 2003), Grupo Scimago (2005, 2006), Jiménez \& otros (2006), Salvador (2007), Jiménez (2007), Cerdán \& otros (2015).

Sobre el impacto de la investigación se han consultado los artículos: "Los mil y un reflejo de las publicaciones en el laberinto de espejos de las nuevas métricas", de Gorraiz (2018) y "Cómo analizar el impacto de los datos de investigación con métricas: modelos y servicios", de Ferrer, Sánchez, Benavent y Peset (2016), y sobre investigación en materias específicas han sido de gran interés los trabajos sobre Comunicación por Caffarel, Ortega y Gaitán (2017), Publicidad por Baladrón Pazos, Mancado y Correyero (2017); Documentación informativa por López y otros (2005), y Documentación periodística por Guallar (2003).

\begin{tabular}{|c|c|c|}
\hline Título & Editor & Fechas \\
\hline Anales de Documentación & Universidad de Murcia & $1998-2019$ \\
\hline BiD. Textos Universitarios & $\begin{array}{l}\text { Facultat de BiD (Universidad de Barcelona) y Estudis de Ciencias de la } \\
\text { Informació i de la Comunicació (Universidad Oberta de Catalunya) }\end{array}$ & 1998-2019 \\
\hline Boletín de Anaban & Asociación de Archiveros, Bibliotecarios, Museólogos y Documentalistas & $1950-2019$ \\
\hline $\begin{array}{l}\text { Cuadernos de Documentación } \\
\text { Multimedia }\end{array}$ & $\begin{array}{l}\text { Departamento de Biblioteconomía y Documentación de la Universidad } \\
\text { Complutense de Madrid }\end{array}$ & $1992-2019$ \\
\hline $\begin{array}{l}\text { Documentación de las Ciencias de } \\
\text { la Información }\end{array}$ & $\begin{array}{l}\text { Departamento de Biblioteconomía y Documentación de la Universidad } \\
\text { Complutense de Madrid }\end{array}$ & $1976-2019$ \\
\hline El Profesional de la Información & Profesional de la Información/ SCP & $1998-2019$ \\
\hline Ibersid & Universidad de Zaragoza & 2007-2019 \\
\hline Item & Col.legi Oficial de Biblioteconomía y Documentación & $1987-2019$ \\
\hline Métodos de información & Colegio Oficial de Biblioteconomía y Documentación (Valencia) & 1998-2002; 2010-2019 \\
\hline $\begin{array}{l}\text { Revista Española de } \\
\text { Documentación Científica }\end{array}$ & Consejo Superior de Investigaciones Científicos/CINDOC & $1977-2019$ \\
\hline $\begin{array}{l}\text { Revista General de Información y } \\
\text { Documentación }\end{array}$ & $\begin{array}{l}\text { Facultad de Ciencias de la Documentación Universidad Complutense de } \\
\text { Madrid }\end{array}$ & $1992-2019$ \\
\hline Scire & Universidad de Zaragoza & $1995-2019$ \\
\hline
\end{tabular}

Tabla I. Revistas de documentación analizadas (2000-2019)

Marcos, J. C.; Sánchez Vigil, J. M.; Olivera Zaldua, M. Producción científica sobre temas educativos y formativos en comunicación, información y documentación en las revistas de documentación españolas. // Ibersid. 14:2 (jul.-dic. 2020) 39-48. ISSN 1888-0967. 


\section{Metodología}

El análisis de las publicaciones y sus contenidos se ha realizado sobre doce revistas de Documentación entre 2000 y 2019 (Tabla I, en la página anterior), tomando como referencia los índices de impacto y su posición desde el año 2000, elaborados por el grupo de investigación EC3 (Evaluación de la Ciencia y de la Comunicación Científica) de la Universidad de Granada, así como el sistema de información RESH (Revistas Españolas de Ciencias Sociales y Humanidades) que integra los indicadores de calidad para las publicaciones científicas españolas, desarrollados por los grupos EPUC (CCHS-CSIC) y el citado EC3. Las publicaciones analizadas tienen una antigüedad superior a los veinte años en los que se enmarca la investigación, es decir que todas fueron creadas antes del 2000, con excepción de Ibersid que salió en 2007, y de Métodos de Información con dos etapas (2000-2002 y 2010-2019).

\subsection{Recogida de datos: generación} de una base de datos

Para obtener la máxima información se han revisado todos los artículos y sus correspondientes sumarios, accediendo desde las webs institucionales. Las consultas específicas se han realizado en las colecciones de las bibliotecas de las Facultades de Ciencias de la Documentación y Ciencias de la Información de la UCM. En los primeros años de algunas publicaciones (Anabad, BiD, Cuadernos de Documentación Multimedia, Item y Métodos de Información) se ha realizado una revisión de los resúmenes y una lectura exhaustiva con el fin de extraer palabras clave. Así mismo, se han recuperado determinados términos que figuran en los títulos y/o resúmenes y no aparecen en las palabras clave.

La recuperación de la información se ha llevado a cabo generando una base de datos diseñada al efecto con los siguientes campos: revista, autor, filiación, artículo, fecha, volumen y número, páginas, medio tratado, tema específico, palabras clave, y observaciones. Una vez procesados los datos y analizados los contenidos, se han obtenido los resultados que se indican a continuación.

\section{Resultados, análisis y evaluación}

Tras el análisis de las revistas se ha obtenido información sobre los autores y su filiación, los centros de investigación, los contenidos, el número de artículos sobre la materia objeto de estudio, las temáticas abordadas y las palabras clave específicas.

\subsection{Producción global y artículos por revistas}

El número total de artículos en las revistas seleccionadas es de 6674 en el periodo estudiado, de los que solo $231(3,47 \%)$ tratan sobre temas educativos y formativos en materia de Comunicación, Información y Documentación, cantidad muy reducida con respecto al total (Tabla II). El año con mayor número de artículos fue 2007 (29), seguido de 2011 (20), 2009 (18) y 2002 (16). De los textos, 36 corresponden a la Revista General de Información y Documentación (15,58 $\%), 31$ a Documentación de las Ciencias de la Información $(13,41 \%)$ y 28 a El Profesional de la Información y Anabad (12,12\%). Las publicaciones con menor número de textos sobre el tema son Métodos de Información (2), Item (4) y Cuadernos de Documentación Multimedia (5).

Al establecer la relación entre el número de artículos por cada revista y el número de textos sobre Educación y formación, los resultados son los siguientes: Documentación de las Ciencias de la Información es la primera, con 31 artículos sobre 278 (11,15\%), Anales de Documentación con 28 sobre 316 (8,86\%), Revista General de Información y Documentación con 36 sobre 450 (8\%) e Ibersid con 18 sobre 293 (6,14\%). El resto se encuentra por debajo del $4 \%$, y no alcanzan el $2 \%$ cuatro revistas: Cuadernos de Documentación Multimedia (1, 44\%), el Profesional de la Información $(1,18 \%)$, Item $(1,50 \%)$ y Métodos de Información (1,04\%) (Tabla III, en la página anterior).

\subsection{Autoría y filiación}

Los autores que escriben sobre la materia en las revistas seleccionados son 336 , de los que tan solo 11 han publicado 4 o más artículos. Tres de éstos autores han escrito 6 textos, y otros tres de ellos 5. En consecuencia, son muchos los autores con uno, dos o tres textos sobre la materia analizada, un alto porcentaje en coautoría.

Los autores con mayor número de trabajos son María Pinto Molina, José López Yepes y Miguel Ángel Marzal, con 6 textos cada uno (los dos primeros con dos individuales cada uno, mientras que en el caso de Marzal todos son en colaboración). Les siguen con 5 textos Carlos Tejada Artigas y Alejandro Uribe Tirado, en ambos casos todos los textos en colaboración excepto uno. Significamos además a Francisco Javier García Marco, con 4 artículos de autoría única (Apéndice I).

El número de autores con un solo artículo es de 123 , por lo que se han tenido en cuenta las coautorías, que presentan los siguientes datos: 55 artículos con dos autores, 29 con tres, 11 con cuatro, 6 con cinco y con seis, y 1 con siete. Se han

Marcos, J. C.; Sánchez Vigil, J. M.; Olivera Zaldua, M. Producción científica sobre temas educativos y formativos en comunicación, información y documentación en las revistas de documentación españolas. // Ibersid. 14:2 (jul.-dic. 2020) 39-48. ISSN 1888-0967. 
considerado como autor único dos grupos de investigación con un artículo cada uno de ellos.

Por lo que respecta a la filiación, son 99 los centros a los que pertenecen los autores, 70 de ellos universidades españolas y extranjeras. El resto (29) se reparten entre instituciones públicas y privadas. Se observa una alta producción en los centros extranjeros (35 de 70 , el $50 \%$ del total) frente a los españoles, tratándose de revistas académicas editadas en España. Los artículos de universidades extranjeras tienen la siguiente procedencia: México (8), Brasil (6), Colombia (5), Chile (4), Ecuador (2), Uruguay (2), Reino Unido (2), Venezuela (1), Cuba (1), Italia (1), Estonia (1), Rumanía (1), Portugal (1) (Tabla IV).

\begin{tabular}{rrrrrrrrrrrrrr}
\hline$A \tilde{n} 0$ & $A N$ & $B A$ & $B I$ & $C D$ & $D C$ & $E P$ & $I B$ & $I T$ & $M I$ & $R E$ & $R G$ & $S C$ & Total \\
\hline 2000 & 1 & 0 & 1 & 1 & 2 & 0 & 0 & 0 & 0 & 0 & 1 & 0 & 6 \\
\hline 2001 & 3 & 0 & 0 & 0 & 2 & 0 & 0 & 0 & 0 & 1 & 2 & 2 & 10 \\
\hline 2002 & 2 & 0 & 1 & 0 & 9 & 1 & 0 & 0 & 0 & 1 & 2 & 0 & 16 \\
\hline 2003 & 0 & 1 & 0 & 0 & 2 & 2 & 0 & 1 & 0 & 1 & 6 & 2 & 15 \\
\hline 2004 & 1 & 0 & 2 & 0 & 2 & 1 & 0 & 0 & 0 & 4 & 0 & 3 & 13 \\
\hline 2005 & 4 & 4 & 0 & 0 & 2 & 0 & 0 & 0 & 0 & 0 & 0 & 0 & 10 \\
\hline 2006 & 1 & 0 & 0 & 0 & 0 & 1 & 0 & 0 & 0 & 0 & 2 & 2 & 8 \\
\hline 2007 & 4 & 6 & 1 & 0 & 1 & 8 & 6 & 0 & 0 & 0 & 3 & 0 & 29 \\
\hline 2008 & 0 & 4 & 0 & 0 & 1 & 2 & 0 & 1 & 0 & 1 & 1 & 0 & 10 \\
\hline 2009 & 2 & 6 & 1 & 1 & 1 & 0 & 2 & 0 & 0 & 2 & 2 & 1 & 18 \\
\hline 2010 & 1 & 0 & 1 & 1 & 2 & 0 & 2 & 1 & 0 & 0 & 1 & 0 & 9 \\
\hline 2011 & 3 & 0 & 0 & 0 & 4 & 2 & 1 & 1 & 0 & 2 & 3 & 4 & 20 \\
\hline 2012 & 1 & 0 & 0 & 0 & 2 & 3 & 1 & 0 & 0 & 4 & 0 & 0 & 11 \\
\hline 2013 & 3 & 0 & 1 & 0 & 0 & 7 & 1 & 0 & 1 & 0 & 1 & 0 & 14 \\
\hline 2014 & 0 & 0 & 0 & 0 & 0 & 0 & 1 & 0 & 0 & 1 & 1 & 0 & 3 \\
\hline 2015 & 0 & 1 & 3 & 1 & 1 & 1 & 1 & 0 & 0 & 0 & 1 & 0 & 8 \\
\hline 2016 & 1 & 1 & 3 & 0 & 0 & 0 & 0 & 0 & 0 & 1 & 4 & 0 & 10 \\
\hline 2017 & 0 & 1 & 0 & 0 & 0 & 0 & 0 & 0 & 0 & 1 & 2 & 0 & 4 \\
\hline 2018 & 0 & 2 & 0 & 0 & 0 & 0 & 2 & 0 & 1 & 1 & 3 & 0 & 9 \\
\hline 2019 & 1 & 0 & 1 & 2 & 0 & 0 & 1 & 0 & 0 & 2 & 1 & 0 & 8 \\
\hline Total & 28 & 26 & 17 & 5 & 31 & 28 & 18 & 4 & 2 & 22 & 36 & 14 & 231 \\
\hline & & & & & & & & & & & & &
\end{tabular}

Tabla II. Artículos por revistas (2000-2019) (Leyendas en tabla siguiente)

\begin{tabular}{lrrr}
\hline Revista & Total & Educación & $\%$ \\
\hline Anales de Documentación (AN) & 316 & 28 & 8,86 \\
\hline Boletín de Anaban (BA) & 709 & 26 & 3,66 \\
\hline BiD (BI) & 436 & 17 & 3,89 \\
\hline Cuadernos de Documentación Multimedia (CD) & 347 & 5 & 1,44 \\
\hline Documentación de las CC. de la Información (DC) & 278 & 31 & 11,15 \\
\hline El Profesional de la Información (EP) & 2364 & 28 & 1,18 \\
\hline Ibersid (IB) & 293 & 18 & 6,14 \\
\hline Item. Revista de Biblioteconomia i Documentación (IT) & 265 & 4 & 1,50 \\
\hline Métodos de Información (MI) & 192 & 2 & 1,04 \\
\hline Revista Española de Documentación Científica (RE) & 659 & 22 & 3,33 \\
\hline Revista General de Información y Documentación (RG) & 450 & 36 & 8,00 \\
\hline Scire (SC) & 365 & 14 & 3,83 \\
\hline Total & 6674 & 231 & 3,46 \\
\hline
\end{tabular}

Tabla III. Artículos totales y artículos sobre educación 


\begin{tabular}{|c|c|c|}
\hline Instituciones, Bibliotecas y Archivos & Universidades & Universidades \\
\hline Academia El Caparazón & Adventista de São Paulo (Brasil) & La Salle (Colombia) \\
\hline Archivo del Arzobispado Mérida-Badajoz & Alcalá & London (Reino Unido) \\
\hline Archivo general de la región de Murcia & Alicante (7) & Málaga \\
\hline Arxiu Municipal de Salou & Antioquia (Colombia, 6) & Murcia (18) \\
\hline Ayuntamiento de Morata de Tajuña & Autónoma Agraria A. Narro (3) & Nacional de Colombia \\
\hline Biblioteca Daniel Cossío Villegas (México) & Autónoma Barcelona (2) & Nacional de Loja (Ecuador) \\
\hline Biblioteca del I.E.S. Beatriz Galindo & Chihuahua (México, 4) & Navarra (2) \\
\hline Biblioteca Municipal Ricardo León de Galapagar & Autónoma Madrid (2) & Oberta de Catalunya (15) \\
\hline Biblioteca Nacional de Colombia & Metropolitana (México, 3) & Politécnica de Catalunya (4) \\
\hline Biblioteca Pública Municipal Santa Badajoz & Nueva León (México, 3) & Politécnica de Cartagena \\
\hline Biblioteca Vasconcelos (México D.F.) & San Luis Potosí (México, 7) & Politécnica de Madrid \\
\hline $\begin{array}{l}\text { Col·legi Oficial de Bibliotecaris i Documentalistes de } \\
\text { Catalunya }\end{array}$ & Barcelona (7) & Politécnica de Valencia (11) \\
\hline Colegio Oficial de Enfermería de Madrid & Brasilia (Brasil) & Pompeu Fabra (6) \\
\hline Conselleria de Educació y Cultura Generalitat Valenciana & Camilo José Cela (2) & Pontificia Católica Sao Paulo (Brasil) \\
\hline Biblioteca Vasconcelos (México D.F.) & Carlos III (30) & Rovira i Virgili (3) \\
\hline Consejo Superior de Investigaciones Científicas (2) & $\begin{array}{l}\text { Central "Marta Abreu "de Las Villas } \\
\text { (Cuba) }\end{array}$ & Salamanca \\
\hline Consultor T.I.C & Complutense de Madrid (25) & Santiago de Chile (2) \\
\hline Cruz Roja Madrid & Córdoba (7) & Santiago de Compostela \\
\hline Estudio de Técnicas Documentales & Del Mar (Oaxaca, México) & Sevilla \\
\hline IFLA & Del Zulia (Venezuela) (4) & Studi di Parma (Italia) \\
\hline Instituto de Desarrollo Comunitario Murcia (3) & Escuela de Enfermería (UCM) & Tallin University (Estonia) \\
\hline Instituto E.S. La Melva & Empresa de Uruguay & $\begin{array}{l}\text { Técnica Particular de Loja } \\
\text { (Escuador) }\end{array}$ \\
\hline Investigadores y especialistas (9) & Estadual de Londrina (Brasil) & Transilvania Brasov (Rumania) \\
\hline Lead4Revenue & Estadual Paulista (Brasil) (8) & Sao Paulo (Brasil) \\
\hline Ministerio de Comunicación de Brasil & Evora (Portugal) & Sergio Arboleda (Colombia) \\
\hline ONCE-CIDAT & Extremadura (11) & UNED (6) \\
\hline Rosenberg Library (EEUU) & Federal Fluminense (Brasil) & UNAM (México) (19) \\
\hline Seminario San Atón & Francisco de Vitoria (2) & Uruguay \\
\hline TV3 & Girona & Valladolid \\
\hline \multirow[t]{7}{*}{ Vass Media } & Granada (22) & Vic (2) \\
\hline & Guadalajara (México) & Viña del Mar (Chile, 2) \\
\hline & Illes Balears & Wales a Aberystwyth (Reino Unido) \\
\hline & Internacional de la Rioja & Zaragoza (11) \\
\hline & \multicolumn{2}{|l|}{ Jaume I de Castellón } \\
\hline & \multicolumn{2}{|l|}{ La Coruña } \\
\hline & La Guajira (Colombia) & \\
\hline
\end{tabular}

Tabla IV. Filiación de los autores

\subsection{Contenidos}

Los enfoques en el tratamiento del tema que nos ocupa son muy diversos, con mayor incidencia en la alfabetización y el aprendizaje, así como los modelos de estudio y su evolución. Las palabras clave son determinantes para conocer resultados, y en los 231 artículos suman 644. Sin embargo, los términos coincidentes son muy pocos por lo que ha sido necesario agruparlos en la mayoría de los casos en bloques genéricos. Es el caso de "Bibliotecas", del que aparecen "bibliotecas universitarias" en 16 ocasiones y "bibliotecas escolares" en 6, mientras que el resto hasta sumar 75 se dispersan. Otro ejemplo sería "Formación", cuyo genérico se recupera en 15 ocasiones y el resto se presenta con adjetivos hasta alcanzar los 48 registros. 
Los términos más empleados suman 475 palabras clave, por lo que el resto (169) aparecen una sola vez. Los más utilizados se distribuyen como se indica: bibliotecas $(75$, generales y especializadas), formación (48), educación (47), enseñanza (46, presencial y a distancia), universidad (34) Alfabetización (40) (Tabla V). Con el fin de estructurar los contenidos, las palabras clave se han agrupado en tres campos generales: alfabetización (aprendizaje, competencias, habilidades), docencia (educación, enseñanza, formación, metodología, recursos) y centros (bibliotecas, universidades).

\begin{tabular}{lc}
\hline Palabras clave & Artículos \\
\hline Alfabetización & 40 \\
\hline Aprendizaje & 24 \\
\hline Bibliotecas & 75 \\
\hline Competencias & 26 \\
\hline Docencia & 12 \\
\hline Documentación & 25 \\
\hline Educación & 47 \\
\hline Enseñanza & 46 \\
\hline Estudios & 23 \\
\hline Evaluación & 22 \\
\hline Formación & 48 \\
\hline Gestión & 14 \\
\hline Información & 17 \\
\hline Metodología & 11 \\
\hline Recursos & 11 \\
\hline Universidad & 34 \\
\hline
\end{tabular}

Tabla V. Palabras clave más utilizadas

\section{Discusiones}

Es significativo que palabras clave de uso habitual en educación y formación, y que forman parte del lenguaje oficial de las publicaciones del Espacio Europeo de Educación Superior, tales como actitud o aptitud, no aparezcan en ningún artículo, y que otras se hayan recogido en menos de 10 artículos. Sirvan como ejemplo: archivos (9), plan de estudios (7), perfil docente (6), Grados (7), habilidades (4), asignaturas (2), conocimiento (2), ética y deontología (5).

Hay que recordar que por norma y obligación la totalidad de los artículos que se publican en revistas de calidad dan mucho valor a las palabras clave y son un referente para que los investigadores entiendan el contenido de un artículo, junto con el resumen. Especialmente resulta llamativa por ambigua, al no especificar, la palabra clave recursos, pues se ha convertido en aporte fundamental para el campo de la educación en todos sus ámbitos.

Es discutible también la escasa participación de autores por encima de los cinco artículos; la mayoría muy por debajo, lo que entendemos viene a significar que los temas no tienen una continuidad.

La hipótesis prevista viene a demostrar que los primeros resultados indican la lejanía entre ambas disciplinas. De ahí la necesidad de buscar puntos comunes, interrelacionar las dos áreas y dar valor a las publicaciones de ambas márgenes, especialmente en lo que respecta a las instituciones educativas. Se debe, por tanto, hacer un llamamiento a los autores para que saquen rendimiento a la relación entre la Documentación y la Educación y las contemplen en sus futuras publicaciones.

\section{Conclusiones}

El avance de las ciencias depende de su interrelación, y más, en disciplinas como la Educación y la Comunicación, que emplean elementos comunes como son la información, los datos, los recursos, etc. Esa simbiosis, hasta ahora escasa en España, no tanto en el ámbito latinoamericano, ha de servir como resorte para iniciar nuevas maneras de enseñar gracias a la comunicación y de aprender por mor de lo que los medios de comunicación publican y la documentación guarda.

Una vez analizados los resultados, se concluye que los artículos sobre Educación y formación en materias de Comunicación, Información y Documentación publicados desde el 2000 en las revistas del área de Documentación son muy escasos en comparación con el total, tan solo el $3,47 \%$, si bien aunque la media sea muy baja destacan en el conjunto por su aportación tres publicaciones: Documentación de las Ciencias de la Información $(11,15 \%)$, Anales de la Documentación $(8,86 \%)$, Revista General de Información y Documentación $(8 \%)$ e Ibersid (6,14\%). En consecuencia, sería necesario dinamizar la investigación en estos temas en las revistas del área; en primer lugar, para un mayor conocimiento y difusión de las problemáticas y sus actuaciones, y en segundo término para contribuir a la visión general de los modelos educativos y formativos del área. Se sugiere como método para llevarlo a cabo la convocatoria y publicación de monográficos por las revistas aquí analizadas.

Se observa un sesgo en la investigación hacia los trabajos relacionados con la formación y los estudios sobre bibliotecas (75 artículos), al contrario que sobre los archivos (9 artículos) y otros centros de documentación en general. En este 
sentido se detectan muy pocas publicaciones en lo que se refiere al análisis de la formación y estudios especializados, como son los de Comunicación, Información y Documentación relacionados con los medios, la medicina, la música y tantos otros de interés.

Desde el punto de vista del análisis documental, indicamos que algunos de los términos que figuran en los títulos de los artículos que se han recogido no se reflejan en las palabras clave, si bien se han recuperado para elaborar el trabajo. Por ello, en aras de conseguir la máxima difusión se deben arbitrar fórmulas por parte de los editores para que se preste una mayor atención a este asunto, de forma que se garantice la recuperación de la información y en consecuencia la visibilidad. Entendemos que en las normas de evaluación de las revistas debe incluirse un punto específico en el que se contemple la revisión de las palabas clave puesto que son un referente para los investigadores a la hora de hacer búsquedas en su trabajo.

Somos conscientes de que al hacer este estudio hemos visto algunas limitaciones; por ejemplo, se ha detectado con frecuencia el uso de términos sinónimos en diferentes artículos para indicar cuestiones similares, lo que genera ruido y confunde al lector/investigador. Se plantea de nuevo aquí la necesidad de normalización, e incluso de elaboración de un listado breve de términos a modo de glosario y/o guía.

Por último, el valor de este trabajo se refiere más a la alerta detectada en el escaso uso de las disciplinas, que tienen tanto en común, y el aviso para que otros investigadores se introduzcan en publicaciones que pondrán en alza lo que la comunicación, la documentación y la educación tienen en común. Retos que ya se lanzan a los editores y responsables de revistas en estas materias.

\section{Referencias}

Altbach, P. G.; Teichler, U. (2001). Internationalization and exchanges in a globalized university. // Journal of Studies in International Education. 5:1

Arquero, Rosario. (2002). Autores más citados en publicaciones periódicas del área de Biblioteconomía y Documentación: España, 1975-1948. // El Profesional de la Información. 11:6, 436-441.

Arquero, Avilés Rosario (2003). Evaluación de la colaboración científica en trabajos difundidos en publicaciones periódicas del área de Biblioteconomía y Documentación: España 1975-1984. // Revista General de Información y Documentación. 13:1, 247-259.

Arquero, Rosario; Río Sadornil, José Luis (2002). Trayectoria y estudio de producción de la revista Documentación de las Ciencias de la Información en su XXV aniversario (1976-2001). // Documentación de las Ciencias de la Información. 25, 95-114.
Baladrón Pazos, Antonio J.; Manchado Pérez, Benajmín; Correyero Ruiz, Beatriz (2017). Estudio bibliométrico sobre la investigación en publicidad en España: temática, investigación, redes y centros de producción. // Revista Española de Documentación Científica, 40:2.

Beaver, Donald Deb. (2001). Reflections on scientific collaboration (and its study): past, present, and future // Scientometrics. 52(3).

Cerdán Medina, José Carlos; Ortega Prieto, José Luis; Rodríguez Yunta, Luis (2015). Caracterización de las revistas Españolas de Biblioteconomía y Documentación: Producción científica y perfil temático en el periodo 2004-2013. Madrid: CSIC-ISOC.

CONACYT (2006). Indicadores de actividades científicas y tecnológicas, México: Edición de bolsillo.

De Fillipo, D. (2013). La producción científica española en Comunicación en WOS. Las revistas indexadas en SSCI (2007-12). // Comunicar: Revista científica iberoamericana de comunicación y educación, 41, 25-34.

Delgado López Cozar, Emilio (2001). Las revistas españolas de Ciencias de la Documentación: productos manifiestamente mejorables. // El Profesional de la Información. 10:12, 46-56.

Eco, Umberto (Coord.) (2015). La Edad Media. I. Bárbaros, cristianos y musulmanes. México: FCE, 171.

Ferrer Sapena, Antonia; Sánchez Pérez, Enrique Alfonso; Benavent Rafael Aleixandre; Peset, Fernanda (2016). Cómo analizar el impacto de los datos de investigación con métricas: modelos y servicios // El profesional de la Información. 25:4, 632-641.

Gómez Fuentes, Héctor (1993). Las revistas latinoamericanas de bibliotecología y ciencias de la información bajo el prisma de los servicios bibliográficos internacionales. // Investigación Bibliotecológica. 14:7, 27-32.

Gorraiz, Juan (2018). Los mil y un reflejo de las publicaciones en el laberinto de espejos de las nuevas métricas // El Profesional de la Información. 27:2, 231-236.

Grupo Scimago (2005). Producción española con visibilidad internacional (ISI-WOS) en biblioteconomía y documentación (I). // El Profesional de la Información. 14:6, 459461.

Grupo Scimago (2006). Producción española con visibilidad internacional (ISI-WOS) en biblioteconomía y documentación (II). // El Profesional de la Información. 15:1, 34-36.

Guallar, Javier (2003). Métodes i tècniques de recerca en els articles de documentació periodística a Espanya (19972002). // BiD. 11. http://bid.ub.edu/11gualla.htm

Guallar, Javier; Ferran, Nuria; Abadal, Ernest; Server, Adán. (2017). Revistas científicas de información y documentación: Análisis temático y metodológico. // El Profesional de la Información. 26:5, 947-960.

Harter, S.P.; Hooten, P.A. (1992). Information science and scientists: JASIS, (1972-1990). // Journal of the American Society for Information Science. 43:9, 583-593.

Herrero Solano, Víctor; Ríos Gómez, Claudia. (2006). Producción latinoamericana en biblioteconomía y documentación en el Social Science Citation Index (SSCI) // Information Research. 11:2.

Jiménez, E.; Delgado, E.; Ruiz, R. (2006). Producción española en biblioteconomía y documentación con visibilidad internacional a través del Web of Science (1995-2004). // El Profesional de la Información. 15:5, 373-383.

Jiménez, S. (2007). Análisis de la autoría en la Revista Española de Documentación Científica (1997-2005) // Revista Española de Documentación Científica. 30:3.

Labaree, D. (2000). On the nature of teaching and teacher education: Difficult practices that look easy. // Journal of Teacher Education. 51:3, 228-233, 305-322.

Marcos, J. C.; Sánchez Vigil, J. M.; Olivera Zaldua, M. Producción científica sobre temas educativos y formativos en comunicación, información y documentación en las revistas de documentación españolas. // Ibersid. 14:2 (jul.-dic. 2020) 39-48. ISSN 1888-0967. 
Lariviére, V.; Lebel, J.; Lemelin, P. (2004). Les recherches collaboratives en sciences humaines: analyse bibliométrique des practiques, Observatoire des sciences et des tecnnologies-CIRST. Montreal.

Laval, C. C.; Weber, L. (2002). Le nouvel ordre éducatif mundial, OMC, Banque mondiale, OCDE, Commission européenne, Paris: Nouveaux Regards-Syllepse.

Licea, J.; Valles, J.; Arévalo, G.; Cervantes, C. (2000). Una visión bibliométrica de la investigación en bibliotecología y ciencia de la información de América Latina y el Caribe. // Revista Española de Documentación Científica. 23(1), 45-53.

López, P.; Díaz, R. A.; Angulo, S.; Giménez, E. (2001). Estudio bibliométrico y de calidad de la revista General de Información y Documentación (1991-2000) // Revista General de Información y Documentación. 11:1, 11-32.

Marteleto, R. M. (1998). Las revistas científicas de Ciencia de la Información. Reflexiones a partir de las condiciones de investigación en Brasil. // Documentación de las Ciencias de la Información. 29:1, 45-54.

Moya, F. d.; Herrero, V. (2002). Visibilidad internacional de la producción científica iberoamericana en biblioteconomía y documentación (1991-2000). // Revista Ciencia da Informacao. 31:3, 54-65.

Nóvoa, A.; DeJong, W. (2003). Educating Europe - An analysis of EU educational policies. // D. Phillips y H. Ertl (Comps.). Implementing European Union Education and

\section{Anexo I: Autores y publicaciones}

Esquema: Apellidos, N.: Revista Año/Volumen Abreviaturas: AN: Anales; BA: Boletín de Anabad; BD: Biblioteconomía i Documentació; CD: Cuadernos de Documentación Multimedia; DC: Documentación de las Ciencias de la Información; EP: El Profesional de la Información; IB: Ibersid; MI: Métodos de Información; RE: Revista Española de Documentación Científica; RG: Revista General de Información y Documentación; SC: Scire.

Abadal, E.: EP 2007/16-4, 2012/21-2; RE 2009/32-4

Adamiz Povedano, N.: RE 2012/35-2

Aguilera Ortega, R.: SC 2011/2

Aguillo, I. F.: EP 2007/16-5

Alcázar Farías, E. K. RE 2012/35-4

Andreu Lorenzo, L.B.: BA 2007/2

Apple, C. IT 2011/55

Arano, S.; Martínez, G.: RE 2011/34-3

Arenas Vargas, M.: AD 2000/3, 2004/7, 2005/8

Arguedas Sanz, R.: EP 2013/22-6

Arquero Avilés, R.: DC 2002/25, 2009/32

Asensi Artiga, V.: SC 2001/2

Atenas Rivera, J.: EP 2012/21-2

Ávila Roca de Togores, M. P.: AD 2009/12

Ayuso Sánchez, M.J.: RG 2007/17-2

Azcárate Aguilar Amat, P.: EP 2008/17-3

Balça Á.: BA 2007/2

Barchéin, M. J.: EP 2007/16-3

Barnes Anco, P. N.: AD 2001/5

Barrera Gómez, J. A.: BD 2016/36

Bas Martín, N.: RG 2006/16-2, 2010/20

Beik, A. BA 2007/2

Bel, N.: RE 2011/34-3

Benito Morales, F.: SC 2001/2

Bergillo, I.: DC 2012/35

Bernier, J. L.: EP 2007/16-3

Blanco Faura, I.: RG 2009/19

Blázquez Ochando, M.: DC 2011/34

Borgoños Martínez, Ma . D.: BA 2008/4

Bornacelly Castro, J. A.: EP 2007/16-5

Borrego, Á.: BD 2015/35

Bracho López, R.: RE 2012/35-2

Bueno Sánchez, Á.: RE 2004/28-2
Training Policy: A comparative study of issues in four member states (pp. 41-72). Dordrecht, Kluwer Academic Publishers.

Osorio, J. (2013). Pedagogía y ética en la construcción de ciudadanía: la formación en valores en la educación comunitaria. Polis.

Perrenoud, P. (1998). La transposition didactique à partir de pratiques: Des savoirs aux compétences. // Revue des Sciences de l'Éducation (Montréal), XXIV (3), 487-514.

Ríos, A. B. (2001). Diez años de investigación en la Revista Española de Documentación Científica (1989-1999). /l Revista Española de Documentación Científica. 24:4, 433-439.

Salvador, J. (2007). Top ten. Diez años de investigación española en biblioteconomía y documentación (1996-2006). Ranking e Índice $\mathrm{H}$ de los 10 autores en activo más citados. // Revista General de Información y Documentación. 17:2, 159-182.

UNESCO (2004). Informe de la Organización de las Naciones Unidas para la Educación, la Ciencia y la Cultura y la Organización de Cooperación y Desarrollo Económicos sobre estadísticas en materia de ciencia y tecnología. E/CN. 3/2004/15, UNESCO-Comisión de Estadística.

Van Vught, F. A. (1992). La calidad de la educación superior en Europa: el siguiente paso. // Universidad futura. 3:8-9, 59-72.

Butera, M. J.: BI 2007/19

Byrne, A. AD: 2005/8

Caldera Morillo, E.: DC 2011/34

Caldera Serano, J.: DC 2012/35, 2015/38

Calva Cabrera, K. D.: RG 2016/26-2

Calva González, J. J.: DC 2004/27, 2003/13-2

Calzada Prado, J.: EP 2013/22-6

Campa Planas, F.: EP 2011/20-6

Cantín Luna, M.: IB 2010/4; AD 2011/14-2

Carpallo Bautista, A.: BA 2018/1; RG 2001/11-1

Casaldàlida, A.: RE 2011/34-3

Castro Martínez, E.: RE 2008/31-2

Chain Navarro, C.: RG 2002/12-2, 2009/19

Chaves Guimaraes, J. A.: IB 2007/1; SC 2004/1

Cobarsi Morales J.: EP 2002/11-4; IT 2008/49, 2010/53

Cobo Serrano, S.: RG 2016/26-1

Codina, L.: DC 2002/25

Colmenero, M. J.: RE 2011/34-2

Contreras Espinosa, R. S.: CD 2009/20, 2010/21

Cordero Ortega, A.: AD 2009/12

Corina Vieira, K : SC 2003/1

Corona Medina, J. L.: AD 2004/7

Cortés Peña, O.: RE 2019/42-2

Cortés Velades, C.: IB 2007/1

Cruz Solís, A. de la: DC 2008/31

Cuevas Cerveró, A : AD 2005/8; RE 2019/42-1

Currás, E.: DC 2002/25

Dal'Evedove, P. R.: SC 2009/2

Delgado Fuentes, E. A.: AD 2010/13

Delgado López-Coraz, E.: AD 2001/4

Díez Carrera, C.: BA 2003/1

Domenech, Á.: EP 2007/16-5

Eguia Gómez, J. L.: CD 2009/20

Escar Hernández, E.: AD 2011/14-2; IB 2010/4

Espinosa Morabet, S.: EP 2011/20-2

Espinosa Rodríguez, J.: IB 2007/1

Estivill, A.: BD 2006/16

Expósito López, J.: RE 2001/24-3

Extremeño, A.: BA 2007/2

Faba-Pérez, C.: IB 2012/6; CD 2019/30; RE 2017/40-4

Farrús, M.: IT 2011/55

Fernández Bajón, M. T.: BA 2008/4, 2009/2; DC 2005/28

Marcos, J. C.; Sánchez Vigil, J. M.; Olivera Zaldua, M. Producción científica sobre temas educativos y formativos en comunicación, información y documentación en las revistas de documentación españolas. // Ibersid. 14:2 (jul.-dic. 2020) 39-48. ISSN 1888-0967. 
Fernández Cano, A.: RE 2001/24-3, 2003/26-2, 2004/28-2 Fernández García, M. I.: DC 2004/28

Fernández Quijada, D.: DC 2012/35

Fernández Ramos, A.: RE 2016/39-2

Fernández Rodríguez, G.: EP 2007/16-3

Ferrada, C.: RG 2018/28-2

Ferrán Ferrer, N.: EP 2012/21-2

Ferrer Sapena, A.: SC 2004/1

Florido Medina, A.: BA 2016/2

Fonseca Rodrigues, M. E.: SC 2006/2

Fujita, M.S.L.: AD 2007/10; IB 2007/1; SC 2009/2

Galina Russell, I: EP 2012/21-2

Galván Martínez, I.: BA 2017/4

Gamarra González, M. I.: RG 2003/13-1

Gamarra Gonzalo, A.: BA 2018/1

García Alsina, M.: IT 2010/53

García Aracil, A.: RE 2008/31-2

García Gutiérrez, A.: EP 2013/22-2

García Llorente, H. J.: AD 2019/22-1

García Marco, F. J.: IB 2007/1; EP 2013/22-6; SC 2004/1, 2011/2

García Orozco, J. F.: DC 2011/34

García Zorita, C.: EP 2013/22-6; 2001/11-1

Garduño Vera, R.: BA 2009/2, 2005/1-2; DC 2003/26

Garrido Lova, J.: RG 2002/12-2

Girlesa Uribe, A.: RE 2012/35-2

Gomes da Silva, C. S.: CD 2019/30

Gómez Domínguez, D.: EP 2007/16-3

Gómez Hernández, J. A.: AD 2002/5; SC 2001/2

González Cervantes, S.: RE 2019/42-1

González Fernández-Villavicencio, N.: BD 2016/36

González Lozano, F.: IB 2018/12-2

González, E.: AD 2005/8

Gratch Lindauer, B.: AD 2006/9

Grupo Crai Complutense de Madrid: BA 2007/2

Grupo de investigación Otlet: RG 2008/18

Guevara Villanueva, A.: RG 2015/25-2

Guisado González, L.: CD 2019/30

Gutiérrez Arenas, P.: RE 2012/35-2

Gutiérrez Blanco, A. I.: RE 2008/31-2

Gutiérrez Chiñas, A.: BA 2009/2; DC 2003/26

Gutiérrez Vargas, M. E.: AD 2002/5

Gutiérrez, P.: RE 2003/26-2

Haro de San Mateo, M. V. de: IB 2011/5

Hernández Lara, A. B.: EP 2011/20-6

Hernández Pérez, T.: SC 2011/2

Hernández Ruiz, A.: BD 2016/37

Hernández Salazar, P.: RG 2007/17-2; SC 2003/2

Hernández-Rabadal, C.: BD 2013/31

Hooland, S. V.: BA 2009/4

Infante Fernández, L.M.: RE 2017/40-4

Izquierdo Alonso, A.M.: DC 2010/33

Izquierdo Alonso, M.: DC 2010/33

Jiménez Fanjul, N. N.: RE 2012/35-2

Jiménez López, Á.: SC 2011/2

Lamarca Langa, G.: AD 2011/14-2; DC 2009/32; EP 2007/16-5; IB 2010/4

Lamolla, L.: IT 2008/49

Lascurain Sánchez, M. L.: RG 2001/11-1

León Moreno, J. A.: DC 2012/35, 2015/38

Liberal Omaechea, S.: RE 2018/41-4

Liberman, S.: RE 2002/25-4

Licea de Arenas, J. : AD 2000/3, 2005/8; MI: 2013/57

Llanas Pont, M.: RG 2000/10-1

Llano Ochoa, J.: RG 2017/27-2

Lledó Carreres, A.: RG: 2019/29-2

Llorens, J.: EP 2007/16-5

Lloret Romero, N.: SC 2004/1

López Alacid, M. P.: AD 2009/12

López Borrull, A.: IT 2010/53

López Carrato, M. del L.: MI 2018/9-17

López Carreño, R.: AD: 2007/10

López Gómez, E.: RG 2016/26-1

López Gómez, P.: DC 2002/25
López Hernández, F: EP 2007/16-2, 2008/17-6

López Lucas, E.: RG 2002/12-2

López Yepes, A.: AD 2007/10; BA 2005/1-2, 2009/2; DC $2002 / 25$

López Yepes, J.: BA 2008/4, 2009/2; DC 2005/28

López, A.: IT 2008/49

López, C.: IT 2011/55

López, Q: IT 2011/55

Lorente Barajas, J. L.: RG 2006/16-1

Lorenzo Lledo, A.: RG 2019/29-2

Lorenzo Lledó, G.: RG 2018/28-1; 2019/29-2

Losada, M: RE 2011/34-3

Lowe, M.: BD 2006/17

Lozano Guzmán, A.: RE 2012/35-4

Luceño Ramos, B.: RE 2018/41-4

Machin-Mastromatteo, J. D.: IB 2018/12-1

Manso Perea, C.: RE 2019/42-1

Marcos Recio, J. C.: BA 2007/2; IB 2014/8, 2015/9

Marcos Treceño, M. J.: RG 2018/28-2

Marques Pedígolo, F.: SC 2009/2

Martín García, R.: EP 2013/22-6

Martín Moreno, C.: RG 2001/11-1

Martín Pena, D.: RG 2016/26-1

Martínez Abad, F.: AD 2019/22-1

Martínez Andaluz, J. A.: EP 2003/12-1

Martínez Arrellano, F. F. E.: BA 2005/1-2

Martínez Comeche, J. A.: 2007/17-1

Martínez González, Y.: IB 2007/1

Martínez Osorio, P.: RE 2009/32-1

Martínez Pellicer, A.: RG 2002/12-2

Martínez Pestaña, M. J.: RG 2013/23-1

Martínez Solana, Y.: AD 2013/16-2

Martínez Trujillo, D.: IT 2003/35

Marzal, M. A.: AD 2016/19-2; BI 2007/19: EP 2008/17-3; RE 2011/34-2; RG 2009/19, 2011/21

Mata, M.L. da: AD 2016/19-2

Mayol, C.:BD 2000/4

Maz Machado, A.: RE 2003/26-2, 2012/35-2

Medeiros Neto, B.: 2011/21

Melgar Estrada, L. M.: EP 2007/16-5

Méndez Rodríguez, E.: BA 2009/4

Meneses Placeres, G.: DC 2011/34

Menezes, P. J.: IB 2007/1

Miguel Alonso, A. de: BA 2008/4

Millán Reyes, A. N.: AD 2011/14-2

Miranda, A.: RG 2011/21

Monroy Baldi, M. E.: IB 2007/1

Mora Orozco, V. M.: BD 2016/36

Morales Becerra, I.: RG 2011/21

Morales López, V: BD 2009/23; DC 2005/28

Moreiro González, J. A.: AD 2001/4; EP 2003/12-1, 2008/17-3

Moreno Pulido, A.: EP 2015/24-5

Moreno Reséndez, A.: AD 2004/7

Muñoz Cañavate, A.: DC 2007/30; RG 2009/19

Muñoz de Solano y Palacios, B.: RG 2003/13-1

Muñoz Muñoz, A. M.: RE 2004/27-4

Murguía Jácquez, L. P.: IB 2018/12-1

Noguera, I.: IT 2011/55

Ocaña Fernández, A.: RE 2004/28-2

Olivera Zaldua, M.: IB 2014/8, 2015/9

Olvera, M. A.: RG 2017/27-2

Ontalba Ruipérez, J. A.: EP 2007/16-5

Orduña Malea, E.: RE 2012/36-3

Orera Orera, L.: AD 2011/14-2; DC 2002/25; EP 2007/16-4; IB 2010/4

Ortega, J. L.: EP 2007/16-5

Ortiz Repiso, V.: BD 2015/35; EP 2013/22-6; RG 2014/24-2

Ortoll Espinet, E.: EP 2004/13-5; IT 2008/49, 2010/53; RE 2004/27-2

Osuna Alarcón, M. R.: BA 2015/2

Pacios Lozano, A. R.: AD 2013/16-2; BD 2016/36; RG 2014/24-2; SC 2011/2

Páez Arancibia, J.: IB 2007/1

Palomares Montero, D.: RE 2008/31-2

Marcos, J. C.; Sánchez Vigil, J. M.; Olivera Zaldua, M. Producción científica sobre temas educativos y formativos en comunicación, información y documentación en las revistas de documentación españolas. // Ibersid. 14:2 (jul.-dic. 2020) 39-48. ISSN 1888-0967. 
Parra Pujante, A.: MI 2013/57

Parra Valero, P.: RE 2011/34-2

Parraguez, R.: RG 2018/28-2

Pastor Sánchez, J. A.: IB 2011/5

Pecharromán Calvo, D.: BA 2018/1

Pedraza García, M.: AD 2013/16-2

Penadés de la Cruz, H.: EP 2007/16-2

Peña Huertas, M. J. de la: BA 2008/4

Peña Ocando, D.: DC 2011/34

Pérez Aliende, M.: IB 2013/7

Pérez Gómez, A.: EP 2007/16-5

Pérez Iglesias, J.: EP 2013/22-2

Pérez Lorenzo, B.: AD 2013/16-2

Pérez Montoro, M.: EP 2012/21-2

Pérez Ortiz, G.: IB 2018/12-2

Pérez Pulido, M.: BD 2004/13; RE 2004/27-3

Pérez Sánchez, A. M.: AD 2009/12

Pérez, G.: EP 2007/16-3

Peset Mancebo, F.: SC 2004/1

Pinto Molina, M.: AD 2012/15-2; IB 2009/3; RE 2009/32-1; SC 2011/2

Pinto Santos, A. R.: RE 2019/42-2

Pinto Zuñiga, R.: RG 2016/26-1

Pirela Morillo, J.: DC 2010/33

Pirnuta, O.-A.: RG 2003/13-1

Ponzoni, P.: BD 2004/13

Popa, S.: RG 2003/13-1

Portillo Fuenmayor, L.: DC 2010/33, 2011/34

Poveda Serra, P.: AD 2009/12

Prat Sedeño, J.: DC 2002/25, 2005/28; IB 2019/13-1; RG 2003/13-2; SC 2006/2

Puertas Valdeiglesias, S.: AD 2012/15-2

Punín Larrea, M. I.: RG 2016/26-2

Puraivan, E.: RG 2018/28-2

Ramos Fandiño, G. P.: BA 2009/2

Ramos Gorospe, M.: SC 2011/2

Ramos Simón L. F.: RG 2007/17-1

Razquin Zazpe, P.: RG 2007/17-1

Reducindo, I.: RG 2017/27-2

Reig-Hernández, D.: EP 2013/22-6

Reyes Barragán, M. J.: BA 2009/2

Rico Callado, F. L.: IB 2012/6

Río Sadornil, J. L.: DC 2000/23, 2002/25

Ríos Hilario, A. B.: AD 2011/14-2; IB 2007/1

Rivera, J.: RG 2017/27-2

Rivera, L. R.: RG 2017/27-2

Rodrigo San Juan, C.: EP 2013/22-6

Rodríguez Conde, M. J.: AD 2019/22-1

Rodríguez Martínez, R.: AD 2004/7

Rodríguez Reséndiz, P. O.: CD 2000/9

Rodríguez Sierra, B.: BA 2009/2

Rodríguez, C.: BD 2002/9

Rojas Sateler, F.: EP 2012/21-2

Romo González, J. R.: IB 2018/12-1

Ros García, J.: DC 2000/23; RG 2003/13-24

Rueda Martínez, I.: BD 2016/36

Russell, J. M.: RE 2002/25-4

Saen de Casas, E.: BD 2016/36
Salaberría Lizarazu, R.: EP 2013/22-2

Sales, D.: RE 2009/32-1

Salgado Orellana, N.: RG 2018/28-2

Salvador Oliván, J. A.: AD 2011/14-2; DC 2002/25, 2009/32; IB 2010/4

Sánchez Ambril, G.: AD 2007/10

Sánchez Fernández, R.: EP 2015/24-5

Sánchez Márquez, C.: BA 2009/2

Sánchez Rebull, M. V.: EP 2011/20-6

Sánchez Santos, M.: AD 2011/14-2

Sánchez Vigil, J. M.: IB 2014/8, 2015/9

Santanarch, F.: IT 2011/55

Santos, G.: BD 2019/43; EP 2012/21-2

Sanz, S.: IT 2008/49

Sanz Casado, E.: RG 2001/11-1

Sanz Martos, S.: EP 2013/22-6

Saorín Pérez, T.: IB 2011/5

Scagliarini Galiano, C.: RG 2018/28-1

Sellers de los Ríos, N.: RG 2011/21

Serra Pallares, M.: EP 2011/20-2

Serrano Vicente, R.: EP 2007/16-4

Sforza Gil, M. del C.: CD 2019/30

Sierra Sánchez, J.: RE 2018/41-4

Silva Casarin, H. de C.: AD 2016/19-2

Somoza Fernández, M.: EP 2007/16-4; RE 2009/32-4

Suárez Muñoz, Á.: BA 2007/2

Suárez Ramírez, S.: BA 2007/2

Tannuri de Oliveira, F.: SC 2004/1

Tapia López, A.: DC 2001/24 (2)

Tarango, J.: IB 2018/12-1

Tejada Artigas, C.: EP 2003/12-1; RG 2002/12-1, 2003/131, 2008/17-3; RG 2007/17-1

Terrasa, A.: EP 2007/16-5

Terrón Torrado, M.: RV 2004/27-3

Torralbo Rodríguez, M.: RE 2003/26-2, 2004/28-2, 2012/35-2

Torres Ramírez, I. de: DC 2002/25

Tuñez López, M.: AD 2013/16-2

Uribe Tirado, A.: BD2010/24; EP 2007/16-5; IB 2010/4; RE 2012/35-2, 2014/37-3

Valadez Olguin, R. G.: IB 2007/1

Vallejo Ruiz, M.: RE 2004/28-2

Varela Prado, C.: IB 2009/3

Velázquez, S.: 2005/8

Vendrell, E.: EP 2007/16-5

Vergueiro, Waldomiro C.S.: EP 2008/17-3

Vianello Osti, M.: AD 2013/16-2; RG 2014/24-2; SC 2011/2

Vidal Bordés, F. J.: AD 2011/14-2; IB 2010/4

Vilches Pardo, J.: BA 2005/1-2

Villanueva Valadez, C.: RE 2019/42-2

Villegas, M.: RE 2011/34-3

Viñao, A.: AD 2005/5

Virkus, S.: BD 2015/35

Vivas Moreno, A.: IB 2018/12-2; RG 2016/26-1

Vives i Gràcia, J.: AD 2005/8

Yarza de los Ríos, V.A.: RG 2017/27-2

Zapata Guerrero, M.: IB 2007/1

Zapatero Lourinho, A. S.: BA 2007/2; DC 2004/28

Zapirain Sagaseta, P. J.: EP 2006/15-1

Marcos, J. C.: Sánchez Vigil, J. M.; Olivera Zaldua, M. Producción científica sobre temas educativos y formativos en comunicación, información y documentación en las revistas de documentación españolas. // Ibersid. 14:2 (jul.-dic. 2020) 39-48. ISSN 1888-0967. 